U.S. Department of the Interior

U.S. Geological Survey

Prepared in cooperation with the

Fenway Alliance,

U.S. Environmental Protection Agency,

Massachusetts Department of Environmental Management,

City of Boston, Department of Parks and Recreation,

Town of Brookline, Department of Public Works

\title{
Channel Morphology and Streambed-Sediment Quality in the Muddy River, Boston and Brookline, Massachusetts, October 1997
}

BY ROBERT F. BREAULT, PETER K. WEISKEL, and TIMOTHY D. MCCOBB

Water-Resources Investigations Report 98-4027
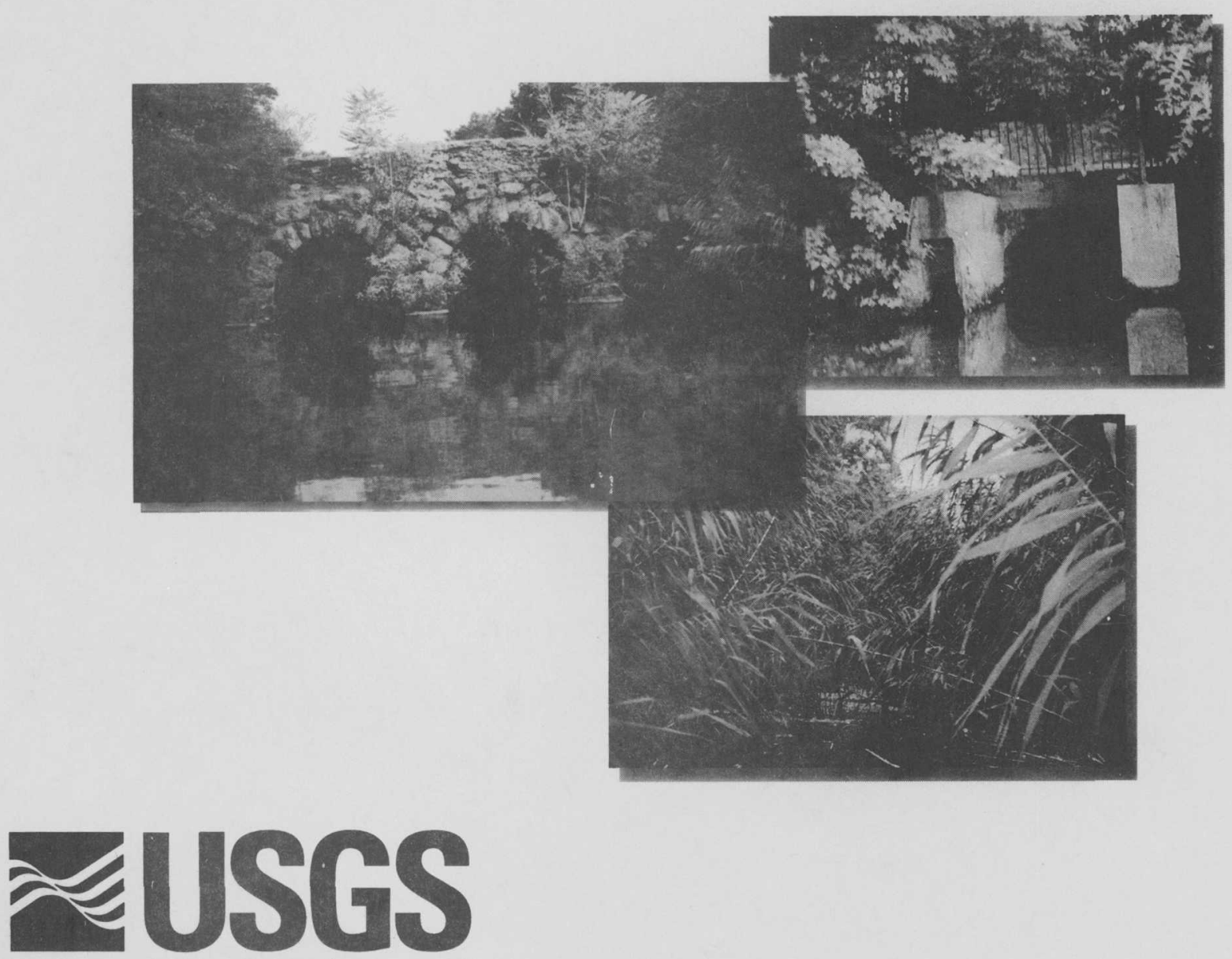

science for a changing world

Marlborough, Massachusetts 
- 
U.S. Department of the Interior

U.S. Geological Survey

Prepared in cooperation with the

Fenway Alliance,

U.S. Environmental Protection Agency,

Massachusetts Department of Environmental Management,

City of Boston, Department of Parks and Recreation,

Town of Brookline, Department of Public Works

Tables to Accompany

Water-Resources Investigations Report 98-4027

\section{Channel Morphology and Streambed-Sediment Quality in the Muddy River, Boston and Brookline, Massachusetts}

By ROBERT F. BREAULT, PETER K. WEISKEL, and TIMOTHY D. McCOBB

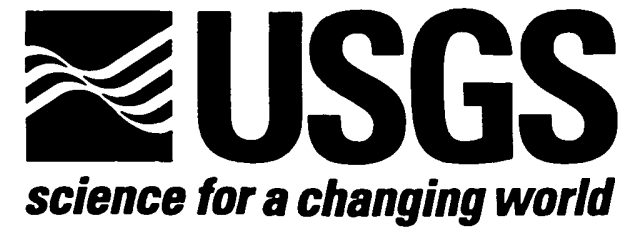

Marlborough, Massachusetts 


\section{SUPPLEMENTAL DATA}

Concentrations of trace metals, organic compounds, total organic carbon, grain-size distributions, percentage of moisture, and toxicity characteristic leaching procedure were analyzed in streambed sediment samples collected at 15 sites in the Muddy River, Massachusetts, in October 1997. These data can be used in conjunction with those presented on the accompanying map report to assist in the design of a remedial program for Muddy River sediments such a program might consider including the advisability of sediment removal, and optimal disposal and re-use options for the dredged sediments.

\section{REFERENCES USED IN SUPPLEMENTAL DATA TABLES}

American Public Health Association, American Water Works Association, and Water Pollution Control Association, 1995, Standard methods for the examination of water and wastewater, 19th ed: Washington, D.C., APHA (variously paginated)

American Society for Testing and Materials, 1980, Natural Building Stones; Soil and Rock: Annual Book of ASTM Standards, part 19, Philadelphia, 634 p.

U.S. Environmental Protection Agency, 1983, Methods for chemical analysis of water and wastes: U.S. Environmental Protection Agency, Cincinnati, Ohio, EPA-600/4-79-020, March 1979, Revised March 1983.

1992, Test methods for evaluating solid waste, physical/chemical methods SW-846, 3rd ed., vol. IC, Chap. 8, sec. 8.4 , rev. 0 , final update, $7 / 92$.

1996, Test methods for evaluating solid waste, physical/chemical methods: SW-846, 3rd ed., Rev. 1 and 2, final update I, 7/92 and final update III, $12 / 96$.

Table 1. Geographic distribution of trace metals and organic compounds in sediment cores, Muddy River, Massachusetts, October 1997

[All concentrations are in parts per million. Trace metals: Analyses were performed in accordance with sample preparation method $3050 \mathrm{~B}$ and ICP method 6010B (U.S. Environmental Protection Agency, 1996, rev. 1 and 2). Mercury: Analyses were performed in accordance with method 245.5 (U.S. Environmental Protection Agency, 1983). Organochlorine pesticides and polychlorinate biphenyls: Sample preparation was done by the EPS MultiMedial Consensus Organics Protocol-Revised 8/87. A macro-Florisil column was used for the sample cleanup. The analysis was carried out using high resolution capillary column chromatography. The 30-m dual capillary system consists of J\&W DB-1701 and J\&W DB-5, both with a 0.25 mm ID and a 0.25 micrometer fil thickness (Peter Philbrook, Office of Measurement and Evaluation Division of the U.S. Environmental Protection Agency, Region I, written commun., 1997). Polyaromatic and total petroleum hydrocarbons: Sample analysis was performed according to EPA Region I Procedure for Polyaromatic Hydrocarbons in Sediment Samples PAHSELL1.SOP. The extracts were analyzed on the gas chromatograph/mass spectrometer using the selected ion monitoring Dick Siscanaw, Office of Measurement and Evaluation Division of the U.S. Environmental Protection Agency, Region I, written commun., 1997) . D, duplicate split sample; No., number; e, estimated; <, less than method detection limit]

\begin{tabular}{|c|c|c|c|c|c|c|c|c|}
\hline \multicolumn{9}{|c|}{ TRACE METALS } \\
\hline Station No. & Arsenic & Cadmium & Chromium & Copper & Lead & Mercury & Nickel & Zine \\
\hline 1 & $<40$ & 3 & 122 & 203 & 919 & 1.4 & 29.3 & 574 \\
\hline 2 & $<60$ & $<2.7$ & 41.3 & 240 & 657 & 1.2 & 29.2 & 527 \\
\hline 3 & $<30$ & 5 & 91.9 & 281 & 1,100 & 2 & 39.6 & 557 \\
\hline 4 & $<30$ & $<2.8$ & 23.1 & 116 & 210 & e6 & 18.4 & 225 \\
\hline 5 & $<20$ & $<2.7$ & 49.5 & 148 & 532 & 1.4 & 24.8 & 276 \\
\hline 5-D & $<25$ & $<3.0$ & 56.7 & 169 & 590 & 1.3 & 27.8 & 310 \\
\hline 6 & $<30$ & 6 & 112 & 448 & 925 & 2.3 & 43.2 & 879 \\
\hline 7 & $<60$ & $<2.6$ & 37.9 & 225 & 360 & 1 & 31.6 & 481 \\
\hline 8 & $<30$ & $<2.8$ & 26.6 & 85.7 & 156 & 1.4 & 15.5 & 220 \\
\hline 9 & $<45$ & 8.3 & 344 & 710 & 1,320 & 6.3 & 70.6 & 1,070 \\
\hline 10 & $<60$ & 14.8 & 182 & 574 & 979 & 3.3 & 59.9 & 982 \\
\hline 11 & $<25$ & 6.9 & 66.3 & 389 & 1,270 & 2.8 & 37.3 & 778 \\
\hline 12 & $<30$ & 8.2 & 101 & 416 & 1,370 & 2.6 & 45.5 & 880 \\
\hline 13 & $<35$ & 8.9 & 78.6 & 478 & 1,410 & 3 & 44.6 & 882 \\
\hline 14 & $<30$ & 7.9 & 87.6 & 387 & 1,360 & 3.2 & 40.1 & 794 \\
\hline 15 & $<50$ & 9.7 & 78.2 & 605 & 1,260 & 2.5 & 44.8 & 965 \\
\hline
\end{tabular}


Table 1. Geographic distribution of trace metals and organic compounds in sediment cores, Muddy River, Massachusetts, October 1997-Continued

ORGANOCHLORINE PESTICIDES

\begin{tabular}{|c|c|c|c|c|c|c|c|c|c|c|c|c|}
\hline $\begin{array}{c}\text { Station } \\
\text { No. }\end{array}$ & Aldrin & $\begin{array}{l}\text { Alpha- } \\
\text { bhe }\end{array}$ & $\begin{array}{l}\text { Beta- } \\
\text { bhe }\end{array}$ & $\begin{array}{l}\text { Delta- } \\
\text { bhe }\end{array}$ & $\begin{array}{l}\text { Delta- } \\
\text { bah }\end{array}$ & $\begin{array}{c}\text { Gamma- } \\
\text { bah }\end{array}$ & $\begin{array}{c}\text { Alpha- } \\
\text { chlordane }\end{array}$ & \multicolumn{2}{|c|}{$\begin{array}{c}\text { Gamma- } \\
\text { e chlordane }\end{array}$} & $\begin{array}{l}\text { Chlor- } \\
\text { dane } \\
\text { (Tech) }\end{array}$ & $\begin{array}{l}\text { "4,4'- } \\
\text { DDD" }\end{array}$ & $" 4,4^{\prime}-D D E^{\prime \prime}$ \\
\hline 1 & $<0.02$ & $<0.02$ & $<0.02$ & $<0.02$ & $<0.02$ & $<0.02$ & 0.038 & \multicolumn{2}{|c|}{0.03} & $<2.0$ & 3.2 & 0.23 \\
\hline 2 & .022 & $<.007$ & $<.007$ & $<.007$ & $<.007$ & $<.007$ & .023 & \multicolumn{2}{|c|}{$<.007$} & $<.7$ & .37 & .16 \\
\hline 3 & $<.01$ & $<.01$ & $<.01$ & $<.01$ & $<.01$ & $<.01$ & .11 & \multicolumn{2}{|c|}{.091} & $<.1$ & 2 & .26 \\
\hline 4 & $<.01$ & $<.01$ & $<.01$ & $<.01$ & $<.01$ & $<.01$ & .035 & \multicolumn{2}{|c|}{.019} & $<.1$ & .56 & .12 \\
\hline 5 & $<.01$ & $<.01$ & $<.01$ & $<.01$ & $<.01$ & $<.01$ & .041 & \multicolumn{2}{|c|}{.035} & $<.1$ & 1.12 & .099 \\
\hline 6 & $<.01$ & $<.01$ & $<.01$ & $<.01$ & $<.01$ & $<.01$ & .12 & \multicolumn{2}{|c|}{.096} & $<.1$ & .5 & .16 \\
\hline 7 & $<.008$ & $<.008$ & $<.008$ & $<.008$ & $<.008$ & $<.008$ & .008 & \multicolumn{2}{|c|}{$<.008$} & $<.8$ & .026 & .011 \\
\hline 8 & $<.003$ & $<.003$ & $<.003$ & $<.003$ & $<.003$ & $<.003$ & .0069 & $<.0$ & & $<.3$ & .046 & .012 \\
\hline 9 & $<.02$ & $<.02$ & $<.02$ & $<.02$ & $<.02$ & $<.02$ & .088 & .0 & & $<.2$ & 1.3 & .38 \\
\hline 10 & $<.01$ & $<.01$ & $<.01$ & $<.01$ & $<.01$ & $<.01$ & .041 & .0 & & $<.1$ & .62 & .31 \\
\hline 11 & $<.01$ & $<.01$ & $<.01$ & $<.01$ & $<.01$ & $<.01$ & .081 & .0 & & $<.1$ & .63 & .17 \\
\hline 12 & $<.02$ & $<.02$ & $<.02$ & $<.02$ & $<.02$ & $<.02$ & .1 & .0 & & $<.2$ & .66 & .19 \\
\hline 13 & $<.02$ & $<.02$ & $<.02$ & $<.02$ & $<.02$ & $<.02$ & .072 & .0 & & $<.2$ & .51 & .16 \\
\hline 14 & .073 & $<.02$ & $<.02$ & $<.02$ & $<.02$ & $<.02$ & .089 & .0 & & $<.2$ & .63 & .18 \\
\hline 14-D & .075 & $<.02$ & $<.02$ & $<.02$ & $<.02$ & $<.02$ & .072 & .0 & & $<.2$ & .53 & .14 \\
\hline 15 & .048 & $<.02$ & $<.02$ & $<.02$ & $<.02$ & $<.02$ & .053 & .0 & & $<.2$ & .29 & .15 \\
\hline & & & & ORGANC & LOR & PESTICI & DES-Conti & tinued & & & & \\
\hline $\begin{array}{c}\text { Station } \\
\text { No. }\end{array}$ & $\begin{array}{l}\text { "4,4'- } \\
\text { DDT" }\end{array}$ & Dieldrin & $\begin{array}{l}\text { Endo- } \\
\text { sulfan I }\end{array}$ & $\begin{array}{l}\text { Endo- } \\
\text { sulfan II }\end{array}$ & $\begin{array}{l}\text { Endo- } \\
\text { sulfan } \\
\text { sulfate }\end{array}$ & Endrin & $\begin{array}{l}\text { Endrin } \\
\text { alde- } \\
\text { hyde }\end{array}$ & $\begin{array}{l}\text { Endrin } \\
\text { ketone }\end{array}$ & $\begin{array}{l}\text { Hepta- } \\
\text { chlor }\end{array}$ & $\begin{array}{c}\text { Hepta- } \\
\text { chlor } \\
\text { epoxide }\end{array}$ & $\begin{array}{l}\text { Methox- } \\
\text { ychlor }\end{array}$ & $\begin{array}{l}\text { Toxa- } \\
\text { phene }\end{array}$ \\
\hline 1 & 0.11 & $<0.2$ & $<0.2$ & $<0.2$ & $<0.2$ & $<0.2$ & $<0.2$ & $<0.2$ & $<0.2$ & $<0.2$ & $<0.2$ & $<2.0$ \\
\hline 2 & .038 & $<.007$ & $<.007$ & $<.007$ & $<.007$ & $<.007$ & $<.007$ & .1 & $<.007$ & $<.007$ & $<.007$ & $<.7$ \\
\hline 3 & .05 & .07 & $<.01$ & $<.01$ & $<.01$ & $<.01$ & $<.01$ & $<.01$ & $<.01$ & $<.01$ & $<.01$ & $<.1$ \\
\hline 4 & .033 & .013 & $<.01$ & $<.01$ & $<.01$ & $<.01$ & $<.01$ & .095 & $<.01$ & $<.01$ & $<.01$ & $<.1$ \\
\hline 5 & .013 & .015 & $<.01$ & $<.01$ & $<.01$ & $<.01$ & $<.01$ & .0093 & $<.01$ & $<.01$ & $<.01$ & $<.1$ \\
\hline 6 & .028 & .039 & $<.01$ & $<.01$ & $<.01$ & $<.01$ & $<.01$ & .037 & $<.01$ & $<.01$ & $<.01$ & $<.1$ \\
\hline 7 & .021 & $<.008$ & $<.008$ & $<.008$ & $<.008$ & $<.008$ & $<.008$ & .046 & $<.008$ & $<.008$ & $<.008$ & $<.8$ \\
\hline 8 & .013 & $<.003$ & $<.003$ & $<.003$ & $<.003$ & $<.003$ & $<.003$ & .043 & $<.003$ & $<.003$ & $<.003$ & $<.3$ \\
\hline 9 & .055 & .036 & $<.02$ & $<.02$ & $<.02$ & $<.02$ & $<.02$ & .056 & $<.02$ & $<.02$ & $<.02$ & $<.2$ \\
\hline 10 & .04 & .015 & .046 & $<.01$ & $<.01$ & .026 & $<.01$ & $<.01$ & $<.01$ & $<.01$ & $<.01$ & $<.1$ \\
\hline 11 & .34 & .05 & $<.01$ & .099 & $<.01$ & $<.01$ & $<.01$ & $<.01$ & $<.01$ & $<.01$ & $<.01$ & $<.1$ \\
\hline 12 & .028 & $<.02$ & $<.02$ & $<.02$ & $<.02$ & $<.02$ & $<.02$ & $<.02$ & $<.02$ & $<.02$ & $<.02$ & $<.2$ \\
\hline 13 & .011 & $<.02$ & $<.02$ & $<.02$ & $<.02$ & $<.02$ & $<.02$ & $<.02$ & $<.02$ & $<.02$ & $<.02$ & $<.2$ \\
\hline 14 & $<.02$ & .057 & $<.02$ & $<.02$ & $<.02$ & $<.02$ & $<.02$ & $<.02$ & $<.02$ & $<.02$ & $<.02$ & $<.2$ \\
\hline 14-D & $<.02$ & .046 & $<.02$ & $<.02$ & $<.02$ & $<.02$ & $<.02$ & $<.02$ & $<.02$ & $<.02$ & $<.02$ & $<.02$ \\
\hline 15 & $<.02$ & $<.02$ & $<.02$ & $<.02$ & $<.02$ & $<.02$ & $<.02$ & $<.02$ & $<.02$ & $<.02$ & $<.02$ & $<.02$ \\
\hline
\end{tabular}


Table 1. Geographic distribution of trace metals and organic compounds in sediment cores, Muddy River, Massachusetts, October 1997-Continued

\begin{tabular}{|c|c|c|c|c|c|c|c|c|c|}
\hline \multirow{2}{*}{$\begin{array}{c}\text { Station } \\
\text { No. }\end{array}$} & \multicolumn{9}{|c|}{ Aroclor } \\
\hline & 1016 & 1221 & 1232 & 1242 & 1248 & 1254 & 1260 & 1262 & 1268 \\
\hline 1 & $<0.04$ & $<0.04$ & $<0.04$ & 0.078 & $<0.04$ & $<0.04$ & 0.17 & $<0.04$ & $<0.04$ \\
\hline 3 & $<.04$ & $<.04$ & $<.04$ & .22 & $<.04$ & $<.04$ & $<.04$ & $<.04$ & $<.04$ \\
\hline 4 & $<.03$ & $<.03$ & $<.03$ & .081 & $<.03$ & $<.03$ & .092 & $<.03$ & $<.03$ \\
\hline 7 & $<.04$ & $<.04$ & $<.04$ & $<.04$ & $<.04$ & $<.04$ & $<.04$ & $<.04$ & $<.04$ \\
\hline 8 & $<.04$ & $<.04$ & $<.04$ & $<.04$ & $<.04$ & .04 & $<.04$ & $<.04$ & $<.04$ \\
\hline 9 & $<.06$ & $<.06$ & $<.06$ & .33 & $<.06$ & 1.3 & .93 & $<.06$ & $<.06$ \\
\hline 10 & $<.06$ & $<.06$ & $<.06$ & .16 & $<.06$ & .77 & .45 & $<.06$ & $<.06$ \\
\hline 11 & $<.04$ & $<.04$ & $<.04$ & .56 & $<.04$ & 1.7 & .71 & $<.04$ & $<.04$ \\
\hline 14-D & $<.04$ & $<.04$ & $<.04$ & .67 & $<.04$ & 1.7 & .6 & $<.04$ & $<.04$ \\
\hline 15 & $<.05$ & $<.05$ & $<.05$ & .43 & $<.05$ & 1.6 & .59 & $<.05$ & $<.05$ \\
\hline
\end{tabular}

\section{POLYAROMATIC HYDROCARBONS}

\begin{tabular}{|c|c|c|c|c|c|c|c|c|c|}
\hline $\begin{array}{c}\text { Station } \\
\text { No. }\end{array}$ & $\begin{array}{l}\text { Acenaph- } \\
\text { thene }\end{array}$ & $\begin{array}{l}\text { Acenaph- } \\
\text { thylene }\end{array}$ & Anthracene & $\begin{array}{c}\text { Benzo (a) } \\
\text { anthracene }\end{array}$ & $\begin{array}{c}\text { Benzo (b) } \\
\text { fluoran- } \\
\text { thene }\end{array}$ & $\begin{array}{c}\text { Benzo (k) } \\
\text { fluoran- } \\
\text { thene }\end{array}$ & $\begin{array}{c}\text { Benzo (a) } \\
\text { Pyrene }\end{array}$ & $\begin{array}{c}\text { Benzo (ghi) } \\
\text { perylene }\end{array}$ & Biphenyl \\
\hline 2 & 1.9 & .53 & 5.5 & 19 & 26 & 8 & 17 & 11 & .087 \\
\hline 3 & 1.2 & .23 & 3.3 & 8.5 & 11 & 4 & 7.8 & 5.1 & .085 \\
\hline 4 & 5.2 & .43 & 9.2 & 24 & 30 & 8.6 & 23 & 13 & .34 \\
\hline 6 & .57 & .23 & 1.6 & 6.1 & 9.5 & 3.6 & 6.3 & 4.9 & $<.084$ \\
\hline 7 & .76 & .48 & 3.3 & 12 & 18 & 6.1 & 11 & 7.9 & .08 \\
\hline 8 & .46 & .39 & 1.7 & 7.9 & 18 & 6.2 & 11 & 8.5 & .059 \\
\hline 9 & 2.9 & .81 & 8 & 18 & 26 & 9.4 & 18 & 13 & .44 \\
\hline 10 & .38 & .2 & .66 & 3.8 & 7.8 & 2.3 & 4.6 & 3.8 & $<.086$ \\
\hline 14-D & 1.5 & .44 & 3.1 & 10 & 16 & 6.2 & 11 & 7.5 & .14 \\
\hline 15 & 1.1 & .32 & 2.3 & 8.5 & 13 & 4.3 & 8.7 & 5.8 & .14 \\
\hline
\end{tabular}


Table 1. Geographic distribution of trace metals and organic compounds in sediment cores, Muddy River, Massachusetts, October 1997-Continued

\begin{tabular}{ccccccccccc}
\hline \multicolumn{10}{c}{ POLYAROMATIC HYDROCARBONS-Continued } \\
\hline $\begin{array}{c}\text { Station } \\
\text { No. }\end{array}$ & Chrysene & $\begin{array}{c}\text { Dibenzo } \\
\text { (a.h) } \\
\text { anthracene }\end{array}$ & $\begin{array}{c}\text { Fluoran- } \\
\text { thene }\end{array}$ & Fluorene & $\begin{array}{c}\text { "Indeon } \\
(\mathbf{1 , 2 , 3 - c d )} \\
\text { Pyrene" }\end{array}$ & $\begin{array}{c}\text { Naph- } \\
\text { thalene }\end{array}$ & $\begin{array}{c}\text { 2-Methyl- } \\
\text { Naptha- } \\
\text { lene }\end{array}$ & $\begin{array}{c}\text { Phenan- } \\
\text { threne }\end{array}$ & Pyrene \\
\hline 1 & 17 & 2.5 & 37 & 2.7 & 11 & 0.53 & 0.56 & 20 & 31 \\
2 & 24 & 3.3 & 55 & 2.8 & 75 & .41 & .34 & 27 & 44 \\
3 & 10 & 1.5 & 22 & 1.6 & 6.3 & .5 & .29 & 14 & 18 \\
4 & 27 & 3.8 & 64 & 4.9 & 16 & 2.7 & 1.6 & 53 & 55 \\
5 & 5.9 & .8 & 12 & .63 & 3.8 & .17 & .13 & 5.6 & 9.8 \\
6 & 8.4 & 1.2 & 16 & .99 & 5.7 & .18 & .43 & 8.2 & 14 \\
7 & 16 & 2.2 & 32 & 1.3 & 10 & .39 & .28 & 11 & 26 \\
8 & 12 & 2.2 & 19 & .72 & 10 & .27 & .172 & 4.9 & 14 \\
9 & 22 & 3 & 49 & 4 & 15 & 3 & 1.3 & 37 & 39 \\
10 & 6.1 & .86 & 10 & .46 & 4.2 & .27 & .22 & 4.3 & 8.5 \\
11 & 14 & 1.9 & 26 & 2.2 & 8.5 & .49 & 1.2 & 20 & 23 \\
12 & 12 & 1.5 & 23 & 1.5 & 7.3 & .3 & .34 & 14 & 20 \\
13 & 12 & 1.5 & 24 & 2 & 7.3 & .59 & .55 & 16 & 21 \\
14 & 10 & 1.5 & 20 & 1.3 & 6.7 & .29 & .43 & 11 & 18 \\
$14-\mathrm{D}$ & 14 & 2 & 25 & 1.9 & 9 & .49 & .51 & 17 & 23 \\
15 & 11 & 1.5 & 21 & 1.5 & 7.1 & .47 & .51 & 13 & 19 \\
\hline
\end{tabular}

Table 2. Geographic distribution of total petroleum hydrocarbon in sediment cores, Muddy River, Massachusetts, October 1997

[Total petroleum hydrocarbons: Concentrations are in parts per million. Standard analyses were performed in accordance with Methods for Chemical Analysis of Water and Wastes (U.S. Environmental Protection Agency, 1983), EPA-600/4/79-020, Test; Methods for Evaluating Solid Waste, SW-846 (U.S. Environmental Protection Agency, 1996), or standard methods for the examination of water and wastewater (American Public Health Association, 1995. D, duplicate split sample; No., number]

\begin{tabular}{cccc}
\hline $\begin{array}{c}\text { Station } \\
\text { No. }\end{array}$ & $\begin{array}{c}\text { Total } \\
\text { petroleum } \\
\text { hydrocarbon }\end{array}$ & $\begin{array}{c}\text { Station } \\
\text { No. }\end{array}$ & $\begin{array}{c}\text { Total } \\
\text { petroleum } \\
\text { hydrocarbon }\end{array}$ \\
\hline 1 & 27,000 & 9 & 28,000 \\
2 & 21,000 & 10 & 19,000 \\
3 & 25,000 & 11 & 22,000 \\
4 & 9,800 & 12 & 32,000 \\
5 & 11,000 & 13 & 34,000 \\
6 & 30,000 & 14 & 34,000 \\
7 & 16,000 & $14-\mathrm{D}$ & 30,000 \\
8 & 4,700 & 15 & 22,000 \\
\hline
\end{tabular}

Table 3. Geographic distribution of total organic carbon in sediment cores, Muddy River, Massachusetts, October 1997

[All concentrations are in parts per million. Total organic carbon: Analyses were performed in accordance with New England Regional Laboratory Standard Operating Procedure (SOP) 14.1 using a Dohrmann DC-190 TOC Analyzer (William J. Andrade, Office of Measurement and Evaluation Division of the U.S. Environmental Protection Agency, Region I, written commun., 1997). D, duplicate split sample; No., number]

\begin{tabular}{cccc}
\hline $\begin{array}{c}\text { Station } \\
\text { No. }\end{array}$ & $\begin{array}{c}\text { Total organic } \\
\text { carbon }\end{array}$ & $\begin{array}{c}\text { Station } \\
\text { No. }\end{array}$ & $\begin{array}{c}\text { Total organic } \\
\text { carbon }\end{array}$ \\
\hline 1 & 93,143 & 9 & 130,185 \\
2 & 86,477 & 10 & 101,172 \\
3 & 86,523 & 11 & 97,130 \\
4 & 70,333 & 12 & 97,282 \\
5 & 49,255 & 13 & 127,389 \\
6 & 105,433 & 14 & 128,548 \\
7 & 70,381 & $14-\mathrm{D}$ & 118,244 \\
8 & 39,100 & 15 & 114,825 \\
\hline
\end{tabular}


Table 4. Geographic distribution of grain size in sediment cores, Muddy River, Massachusetts, October 1997

[Grain-analysis was performed using ASTM Method D422-63 (American Society for Testing and Materials, 1980). Gravel: sieve number 4, greater than 4.75 millimeters. Coarse sand: Sieve number 10, less than 4.75 millimeters, greater than 2 millimeters. Medium sand: sieve number 40 , less than $\mathbf{2 . 0}$ millimeters, greater than $\mathbf{0 . 4 2 5}$ millimeter. Fine sand: sieve number 200 , less than 0.425 millimeter, greater than 0.075 millimeter. Silt and clay: less than 0.075 millimeter. D, duplicate split sample; No., number]

\begin{tabular}{cccccc}
\hline & \multicolumn{5}{c}{ Grain size (as percentage retained) } \\
\cline { 2 - 6 } $\begin{array}{c}\text { Station } \\
\text { No. }\end{array}$ & Gravel & $\begin{array}{c}\text { Coarse } \\
\text { sand }\end{array}$ & $\begin{array}{c}\text { Medium } \\
\text { sand }\end{array}$ & $\begin{array}{c}\text { Fine } \\
\text { sand }\end{array}$ & $\begin{array}{c}\text { Silt } \\
\text { and } \\
\text { clay }\end{array}$ \\
\hline 1 & 0 & 0.4 & 12 & 28.1 & 57.4 \\
2 & 1.3 & 8.67 & 29.3 & 23.4 & 37.4 \\
3 & .2 & .4 & 10 & 37.7 & 51.7 \\
4 & 1.9 & 6.6 & 34.7 & 33.5 & 23.3 \\
5 & 2.1 & 7 & 24.5 & 39.7 & 26.7 \\
6 & .1 & 1.2 & 26.8 & 23.7 & 48.2 \\
7 & .4 & 5.4 & 31.6 & 24 & 61.4 \\
8 & 6.4 & 11.5 & 44.6 & 22.9 & 14.6 \\
9 & 0 & 4.1 & 31.1 & 22.2 & 42.6 \\
10 & .1 & 2.9 & 28.2 & 25.1 & 43.7 \\
11 & 8 & .2 & 10.1 & 60.3 & 29.4 \\
12 & .3 & 1.5 & 15.9 & 46 & 36.3 \\
13 & 0 & 2.3 & 11.2 & 26.5 & 60 \\
14 & 0 & 2.2 & 16.6 & 30.3 & 50.9 \\
$14-\mathrm{D}$ & 0 & .5 & 16.3 & 31.2 & 52 \\
15 & 0 & .2 & 18.3 & 23.8 & 42.9 \\
\hline
\end{tabular}

Table 5. Geographic distribution of percentage of moisture in sediment cores, Muddy River, Massachusetts, October 1997

[D, duplicate split sample; No., number]

\begin{tabular}{cccc}
\hline $\begin{array}{c}\text { Station } \\
\text { No. }\end{array}$ & $\begin{array}{c}\text { Percentage of } \\
\text { moisture }\end{array}$ & $\begin{array}{c}\text { Station } \\
\text { No. }\end{array}$ & $\begin{array}{c}\text { Percentage of } \\
\text { moisture }\end{array}$ \\
\hline 1 & 58 & 9 & 73 \\
2 & 56 & 10 & 71 \\
3 & 56 & 11 & 54 \\
4 & 43 & 12 & 61 \\
5 & 45 & 13 & 64 \\
6 & 70 & 14 & 58 \\
7 & 58 & $14-\mathrm{D}$ & 59 \\
8 & 50 & 15 & 40 \\
\hline
\end{tabular}

Table 6. Geographic distribution of Toxicity Characteristic Leaching Procedure constituents in sediment cores, Muddy River, Massachusetts, October 1997

[Trace metals: Concentrations are in parts per million. TCLP was performed in accordance with method 1311 (U.S. Environmental Protection Agency, 1992). Sample preparation method 3010A and ICP method 6010B (U.S. Environmental Protection Agency 1996). Mercury: Only samples with a total mercury concentration of about $4 \mathrm{ppm}$ or greater were analyzed. TCLP was perfomred in accordance with method 7470A (U.S. Environmental Protection Agency, 1996). D, duplicate split sample; No., number; <, less than method detection limit; --, not sampled]

\begin{tabular}{|c|c|c|c|c|c|c|c|c|}
\hline $\begin{array}{c}\text { Station } \\
\text { No. }\end{array}$ & Silver & Arsenic & Barium & Cadmium & Chromium & Mercury & Lead & Selenium \\
\hline 1 & $<0.06$ & $<0.25$ & 0.43 & $<0.06$ & $<0.03$ & -- & 1.1 & $<0.20$ \\
\hline 2 & $<.06$ & $<.25$ & .56 & $<.06$ & $<.03$ & -- & 1.4 & $<.20$ \\
\hline 3 & $<.06$ & $<.25$ & .54 & $<.06$ & $<.03$ & -- & 2.2 & $<.20$ \\
\hline 4 & $<.06$ & $<.25$ & .4 & $<.06$ & $<.03$ & $<0.50$ & .76 & $<.20$ \\
\hline 5 & $<.06$ & $<.25$ & .44 & $<.06$ & $<.03$ & -. & 1.7 & $<.20$ \\
\hline 5-D & $<.06$ & $<.25$ & .47 & $<.06$ & $<.03$ & -- & 1.8 & $<.20$ \\
\hline 6 & $<.06$ & $<.25$ & .5 & $<.06$ & $<.03$ & - & .79 & $<.20$ \\
\hline 7 & $<.06$ & $<.25$ & .45 & $<.06$ & $<.03$ & -. & .28 & $<.20$ \\
\hline 8 & $<.06$ & $<.25$ & .29 & $<.06$ & $<.03$ & -. & .7 & $<.20$ \\
\hline 9 & $<.06$ & $<.25$ & .53 & .06 & $<.03$ & $<.50$ & 1.8 & $<.20$ \\
\hline 10 & $<.06$ & $<.25$ & .45 & .14 & $<.03$ & $<.50$ & .87 & $<.20$ \\
\hline 11 & $<.06$ & $<.25$ & .46 & $<.06$ & $<.03$ & -- & 1.2 & $<.20$ \\
\hline 12 & $<.06$ & $<.25$ & .5 & $<.06$ & $<.03$ & -- & 1.4 & $<.20$ \\
\hline 13 & $<.06$ & $<.25$ & .51 & $<.06$ & $<.03$ & -- & 1.3 & $<.20$ \\
\hline 14 & $<.06$ & $<.25$ & .61 & $<.08$ & $<.03$ & -- & 2.9 & $<.20$ \\
\hline 15 & $<.06$ & $<.25$ & .64 & $<.10$ & $<.03$ & -- & 2.8 & $<.20$ \\
\hline
\end{tabular}

